\title{
Edna Hindie Lemay (1928-2006)
}

Annie Duprat, Yann Fauchois, Bernard Gainot, Vivian Gruder et Jean-Pierre Jessenne

\section{(2) OpenEdition}

\section{Journals}

Édition électronique

URL : https://journals.openedition.org/ahrf/7923

DOI : 10.4000/ahrf.7923

ISSN : $1952-403 X$

Éditeur :

Armand Colin, Société des études robespierristes

Édition imprimée

Date de publication : 1 décembre 2006

Pagination : 145-149

ISSN : 0003-4436

Référence électronique

Annie Duprat, Yann Fauchois, Bernard Gainot, Vivian Gruder et Jean-Pierre Jessenne, « Edna Hindie Lemay (1928-2006) », Annales historiques de la Révolution française [En ligne], 346 | Octobre/Décembre 2006, mis en ligne le 01 décembre 2009, consulté le 28 juin 2022. URL : http:// journals.openedition.org/ahrf/7923; DOI : https://doi.org/10.4000/ahrf.7923

Ce document a été généré automatiquement le 24 avril 2022

Tous droits réservés 


\title{
Edna Hindie Lemay (1928-2006)
}

\author{
Annie Duprat, Yann Fauchois, Bernard Gainot, Vivian Gruder et Jean-Pierre \\ Jessenne
}

1 Sur les conseils de Pascal Dupuy, Marc Doray, étudiant à l'Université de Rouen, a pris contact avec Edna Lemay, dans le courant du mois d'avril 2006, au sujet de deux de ses articles publiés aux États-Unis. Gravement atteinte du mal qui l'emportera le 22 juin suivant et souffrant sans cesse, Edna n'a pas hésité : fouillant dans ses dossiers, elle a retrouvé les articles, les a photocopiés et les a immédiatement envoyés à Marc Doray; à la lettre de remerciements que celui-ci lui a adressée, Edna répond encore, dans un courrier en date du 30 mai, pour lui proposer de passer chez elle afin de discuter de ses travaux et voir si elle pouvait l'aider davantage encore. Discrète, Edna était attentive aux plus jeunes et se désolait de constater la perte de la transmission des savoirs dans un monde saisi par l'immédiateté. Chacun des signataires de ce texte a désiré rapporter le lien personnel qui l'unissait à Edna, "citoyenne du monde " autant par ses origines que par ses affections, comme le rappelle Jean-Pierre Jessenne avec qui elle a édité la correspondance des Lepoutre, témoignage à l'inverse d'une famille de fermiers bien ancrée dans le terroir du Ferrain. Pour ce faire, il fallut convaincre les détenteurs des lettres originales, qui ont accepté de collaborer à une opération scientifique non lucrative : la persévérance et la force de conviction d'Edna ont fait merveille en ce genre d'entreprise. Elle entretenait des relations privilégiées avec Yann Fauchois auquel elle adressait de très belles correspondances dont il extrait ici quelques fragments. Pareilles complicités intellectuelles avec Annie Duprat et Bernard Gainot se sont doublées d'une profonde amitié comme en témoignent les dédicaces, toujours très personnelles, qu'Edna portait sur ses ouvrages lorsqu'elle nous les offrait. Que l'écriture à plusieurs de cet hommage soit une manière de célébrer l'humanité et l'amitié d'une authentique historienne de la Révolution et d'une personnalité rare.

2 «Je suis rentrée de Budapest le 16 déc. et, sous l'effet d'un mauvais rhume, je fais la grande paresseuse. Je suis en train d'éliminer les dossiers constitués pour le Dict., ne gardant que les plus parlants. Je viens de terminer un compte-rendu du livre (traduction anglaise) de Baczko sur la Terreur. Et j'irai écouter Furet terminer sur Tocqueville. C'est délicieux de ne plus avoir des obligations de travail !». Edna, qui écrit à Yann Fauchois, est alors [1994] une jeune retraitée... Le ton est plus mélancolique 
quelques années plus tard: «La grande activité que tu sembles admirer n'est que la lâcheté pour cacher une immense solitude. Avant la retraite, je rêvais de faire autre chose et voilà que je suis en train de faire pareil. M'ennuyer dans les bibliothèques pour essayer d'écrire des communications qui n'intéressent pas grand monde. Enfin, la vie, c'est un combat avec soi-même ». On retrouve dans ces deux passages bien des traits d'Edna : la modestie, la force de sa volonté (alors même qu'elle critiquait les méfaits de la politique de la volonté qu'elle voyait à l'œuvre pendant la Révolution française), son obstination (Edna était très têtue), sa franchise voire sa rudesse (envers elle-même et ses proches), ses valeurs (le travail, l'amitié et la fidélité). Au cours d'un de ses derniers dîners avec Annie Duprat et David et Peggy Bien, elle s'emportait encore contre les discours misérabilistes sur le chômage: "quand on veut travailler, on trouve du travail ", ne cessait-elle de répéter. Il est vrai que, bien que diplômée de l'Université de Columbia, elle devient secrétaire de Claude Lévi-Strauss à l'Unesco lorsque les nécessités familiales l'avaient conduite à Paris avec ses enfants; l'anthropologue la remarque (il salue son travail dans ses Mythologiques) et la recommande à Fernand Braudel pour devenir chef de travaux à l'EHESS. Ce travail est aussi une manière de s'inscrire dans un lieu d'échanges intellectuels sans abandonner la passion des voyages et de la découverte de toutes les cultures qui la caractérisait car, née au Caire en 1928 d'un père Arménien réfugié d'Alep et d'une mère américaine, ayant vécu enfance puis études entre Égypte et États-Unis, avec de multiples séjours en Angleterre, Italie ou Suisse, Edna " était » le XX ${ }^{e}$ siècle. Par sa famille et son cheminement personnel, par les situations et les comportements collectifs auxquels elle a été confrontée sont peut-être venus le souci d'authenticité et l'horreur de l'intransigeance dans les jugements politiques qui la caractérisait; nul doute en tout cas, que ce cosmopolitisme vécu a façonné son aptitude extraordinaire à accueillir et faire se rencontrer des gens de partout. Au cours des années 1970, Edna Lemay emmène parfois ses enfants, encore petits, dans des congrès internationaux : en 1979, au Congrès des Lumières à Pise, elle fait la connaissance de Vivian Gruder et se lie d'une amitié pérenne avec elle.

3 Sous l'influence de François Furet dont elle suivait tous les séminaires, c'est l'histoire de la Révolution française, et particulièrement celle des députés aux États généraux, qui retient l'attention de la citoyenne du monde devenue française. La portée universaliste de l'expérience révolutionnaire a certainement joué dans ce choix. Sa thèse - soutenue à l'Université Paris IV au début des années 1970 sous la direction d'Alphonse Dupront, jamais publiée mais souvent pillée - était consacrée à la naissance de l'anthropologie culturelle moderne à travers le futur député Démeunier; elle a été le point de départ d'un travail considérable qui aboutira au Dictionnaire des Constituants. Pour conduire l'aventure de ce dictionnaire, Edna bataille ferme avec l'EHESS pour obtenir des crédits, réunit une petite équipe d'ingénieurs de recherche (Christine Favre-Lejeune, Marie-Laurence Netter, Jean-Louis Ormières) et deux jeunes chercheurs bénévoles (Yann Fauchois et Joël Félix). Travail collectif d'une rigueur scientifique exemplaire, ce dictionnaire a permis la révision de nombreux éléments biographiques approximatifs et la mise au clair de tous les discours des députés de 1789 à 1791 ; célébrons l'utilité d'un ouvrage qui est l'un des plus utilisés par la communauté scientifique des chercheurs sur la Révolution française même si beaucoup oublient d'y faire référence. Spécialiste incontestée des députés de la Constituante, Edna Lemay s'inscrivait dans une historiographie érudite, hors des querelles qui pourtant n'ont cessé de faire rage dans les rangs des spécialistes de la Révolution et sa contribution à notre connaissance de l'action politique des élites révolutionnaires se marque par son 
intérêt pour la vie quotidienne des individus et de leurs itinéraires singuliers et pour la prosopographie, la mise en relation de ces individus et la mise en évidence des réseaux auxquels ils se rapportent, dans un dépassement de l'histoire sociale traditionnelle par le culturel; sensible aux métissages sociaux et culturels mais refusant toute prédétermination des cadres d'analyse holistes ou d'une histoire des idées située dans un pur éther, Edna publie un remarquable article dans la Revue d'histoire moderne et contemporaine en 1977, "La composition du tiers état de l'Assemblée nationale constituante: les hommes de la continuité?». L'expérience du dictionnaire a également nourri les réflexions d'un article publié dans les Annales historiques de la Révolution française en 1991, "Les révélations d'un dictionnaire: du nouveau sur la composition de l'Assemblée nationale constituante» et d'un livre écrit avec la collaboration d'Alison Patrick et de Joël Félix, Revolutionaries at Work. The Constituent Assembly 1789-1791, Oxford, Voltaire Foundation.

Edna aimait à faire se rencontrer des historiens d'horizons souvent ennemis, de l'IHRF ou de l'EHESS car, avec modestie et indépendance d'esprit, elle butinait et récoltait ses moissons sans se soucier autrement des bordées que s'envoyait l'artillerie lourde des deux principaux camps en présence. Elle pouvait ainsi fréquenter des lieux aussi « mal famés » (comme on disait dans son École) sans pour autant concéder quoi que ce soit de ses opinions et en toute fidélité à François Furet. Elle nourrissait surtout l'espoir, par les contacts humains, de jeter des ponts entre les différentes écoles de la Révolution française. Modérée dans l'âme, elle espérait parvenir au compromis par la discussion libre. En cela aussi, elle a joué un rôle dans l'évolution de l'historiographie révolutionnaire, aidant à sa décrispation et préfigurant le paysage de l'après Bicentenaire. Dans les années 1980, Bernard Gainot, alors doctorant à l'IHRF, a fait partie du petit cercle qui fréquentait régulièrement son séminaire à l'EHESS, séduit par l'atmosphère intime et érudite tout à la fois qu'Edna avait su imprimer à ces rencontres avec un public fidélisé. Par la suite, une collaboration s'est approfondie, lorsqu'elle a organisé deux ateliers dans le cadre de l'International Society for the Study of European Ideas (Graz en 1994 et Utrecht en 1998). Chacun a son souvenir personnel de sa première rencontre avec Edna : pour Annie Duprat, qui habitait à deux rues de chez elle, c'est un long trajet en métro pour se rendre au colloque de St-Denis, St-Denis ou le dernier jugement des rois en 1989. Pour tous, après ces premiers contacts et ces discussions savantes, venait le temps de l'amitié et des petits dîners rue des Hospitalières Saint-Gervais. On y parlait de tout, de la Révolution bien sûr, mais aussi de problèmes d'actualité, de la société américaine, de la Palestine, de ses souvenirs de jeunesse, en particulier à Alexandrie, de ses nombreux voyages (elle appréciait particulièrement les Baléares), et aussi, et beaucoup, de la famille.

5 Edna disait être fascinée par la sociabilité des salons du XVIII ${ }^{\mathrm{e}}$ siècle et nourrir le dessein d'en reconstituer quelque chose, à sa modeste échelle. Son domicile était régulièrement fréquenté par nombre de collègues étrangers et ce rôle de passeur qu'elle affectionnait s'incarnait dans son salon. Très fidèle en cela aux idéaux cosmopolites de ce siècle des Lumières qu'elle fréquentait depuis si longtemps, elle adorait recevoir chez elle, mettre en contact les gens d'origine et de génération très diverses avec lesquels elle avait sympathisé au gré de ses pérégrinations, favorisant leurs échanges en disparaissant de longs moments dans une cuisine où seuls quelques rares avaient le privilège de pouvoir l'y aider... C'est avec nostalgie et bonheur que nous repensons à ces innombrables dîners qui mêlaient cette pluralité d'univers auxquels Edna était liée: Amérique, France, Europe de l'Est, Proche-Orient... Programmés ou 
impromptus, ces dîners manqueront à tous ses amis qui trouvaient là une occasion d'échanger hors de toute contrainte représentative. Saluons par ces quelques lignes David et Peggy Bien, Harvey Chisick, William Doyle, Ouzi Elyada, Beatrice Fink, Robert Griffiths, Vivian Gruder et Carlo Poni, Ilona Kovacs, Mila Pimenova, Gabriella Vidan, Silvio et Ruth Yeschua, Eva Ring, Olga Penke, Vladislava Sergienko, Martin Staum, Tim Tackett et Helen Chenut et tant d'autres... Elle évoquait souvent ses plus fidèles amis, Marianne Mahn-Lot, Jean-Louis et Maria Flandrin, Ivo Vidan, déjà disparus. Au fil des ans, ces réunions pouvaient prendre le visage d'une agréable tradition qui rythmait sa vie comme ces colloques où elle courrait et où ses contributions étaient toujours informées et marquées du sceau de l'honnêteté intellectuelle; ses prises de parole d'une totale franchise en ont désarçonné plus d'un.

6 Au début de la présente décennie, mûrit son projet d'un Dictionnaire des Législateurs, qui devait être la grande œuvre de la fin de sa vie; surmontant avec elle les difficultés techniques qui venaient de l'utilisation obstinée d'un matériel informatique un peu obsolète, nous eûmes plusieurs séances de travail qui consistaient en une relecture ligne à ligne, mot à mot, de toutes les fiches accumulées par ses collaborateurs. Mais, malgré l'énergie dont elle ne cessait de faire preuve, Edna n'a pas pu en voir la publication car l'ouvrage a été ralenti par des vents contraires (retards des auteurs, mauvaises transcriptions, défaut de financement...). Infatigable, elle a mûri un autre projet, qui devait être son travail ultime ; une biographie intellectuelle de Démeunier qui s'annonçait comme l'itinéraire d'un homme des Lumières en Révolution, puis en notabilité ; elle souhaitait, en une sorte de boucle, reprendre ses notes et achever ce qu'elle avait commencé dans sa jeunesse. Le destin l'a rattrapée avant que la boucle ne se referme; formulons le souhait que cette œuvre inachevée puisse faire l'objet d'une publication. 